\title{
Less specific recovery strategy targets for threatened and non-charismatic species at risk in Canada
}

\author{
Elysabeth Théberge and Joseph J. Nocera
}

\begin{abstract}
Recovery strategies for species at risk have been criticized for a lack of specificity (i.e. measurable and quantifiable criteria) as well as for taxonomic biases, both of which may ultimately affect species' recovery. However, it is unknown whether the clarity and specificity of written statements within recovery strategies can also influence recovery efforts for certain species at risk. To assess this we examined the variation in semantic uncertainty in the target statements of recovery strategies for Canadian species at risk at the federal and provincial levels. We quantified the lexical density and readability of recovery target statements and examined them for differences according to taxonomic grouping, jurisdiction and degree of endangerment. Recovery statements for the category threatened species had greater semantic uncertainty than those for higher (endangered) and lower (special concern) categories, which is likely to be a function of the fact that threatened species are less abundant than special concern species but are subject to greater errors in population estimates than endangered species. We also found that recovery statements for non-charismatic species (e.g. plants and invertebrates) had greater semantic uncertainty than those for other taxa, which may be related to the resources available for studying and conserving them. Our results suggest a need for greater specificity in recovery targets for threatened and noncharismatic species, and that more focused data collection on these species' populations is warranted.
\end{abstract}

Keywords Canada, population targets, recovery goals, recovery objectives, recovery strategy, semantic uncertainty, threatened species

\section{Introduction}

Tn Canada the federal Species at Risk Act has been implemented to protect imperilled species. Most Canadian provinces also have their own similar legislation, such as Ontario's Endangered Species Act. To protect and recover at-risk species both federal and provincial legislation

Elysabeth ThéBERge Biology Department, Trent University, Peterborough, Ontario, Canada

JOSEPH J. NOCERA (Corresponding author) Ontario Ministry of Natural Resources, Trent University, Peterborough, Ontario K9J7B8 Canada. E-mail joe.nocera@ontario.ca

Received 11 August 2012. Revision requested 5 October 2012.

Accepted 10 October 2012. First published online 31 January 2014. require a recovery strategy for listed species, with criteria and targets that must be met to achieve downlisting. These recovery strategies are important tools in guiding agencies and decision-makers in the conservation and recovery of species at risk. Nonetheless, several aspects of recovery strategies have been criticized as needing improvement (Boersma et al., 2001; Gerber \& Hatch, 2002).

Although recovery strategies are critical for directing conservation activities they often lack key details about population status and threats, or fail to set specific recovery goals (Wilcove et al., 1993), which can make it difficult to determine whether recovery has been successful (Findlay et al., 2009; Mooers et al., 2010). For example, setting a target that a population of blue whales Balaenoptera musculus must 'reach a level of 1,000 mature individuals' (Species at Risk Registry, 2010) before the species can be downlisted provides a firm numeric goal by which progress can be measured by assessing population size over time. In contrast, recovery progress is difficult or impossible to assess when a target criterion simply requires the population to be 'restored and maintained at self-sustaining populations within the species' historic range', as it does for the Banff Springs snail Physella johnsoni (Species at Risk Registry, 2010). Explicit criteria and objectives in recovery plans can include information such as target population size (Elphick et al., 2001), time needed for a species to be considered recovered when a stable population is achieved (Schultz \& Gerber, 2002), and spatially explicit guidelines that include elements such as size of a desired protected habitat (Semlitsch, 2001). For example, the population objective for Henslow's sparrow Ammodramus henslowii requires the establishment of at least one $>_{50}$ ha patch of suitable grassland in Ontario (Species at Risk Registry, 2010). To improve the status of a listed species Schwartz (2008) points out that having an approved recovery strategy with a specific means to track progress will increase the chances of funding, yet such specificity is often absent.

Recovery strategies are often subject to an apparent taxonomic bias towards high-profile vertebrates (Wilcove et al., 1993; Lundquist et al., 2002; Schwartz, 2008) and more detail is provided in recovery strategies for charismatic, economically important or hunted species than those for most plants or insects (Lundquist et al., 2002; Schwartz, 2008). This may be in part a result of value-based sociological or political pressure that focuses the attention of researchers and funding agencies on these species (Schwartz, 2008; Findlay et al., 2009; Mooers et al., 2010). A focus on charismatic taxa may prove beneficial if they 
serve as umbrella species. However, whereas the less charismatic (and typically smaller-bodied) taxa may benefit from the development of recovery strategies for umbrella species, they themselves often lack specific and measurable recovery recommendations (Lundquist et al., 2002).

Some common examples of non-specificity in recovery strategies are objectives and goals written with a high degree of semantic uncertainty, in which statements lack concrete meaning in an ecological sense (e.g. phrases using descriptors such as 'extreme' or 'frequently'; Akçakaya et al., 2000). Although some semantically uncertain statements are irreducible because of limitations in data availability or quality, they generally reduce clarity and specificity for the reader and should be avoided whenever possible (Akçakaya et al., 2000). Even small changes in statements, such as switching verb tense, can affect the inferences readers may draw from them. For example, in exploring the effects of word choice on political re-election potential, Fausey \& Matlock (2011) found that senators who were described as having performed negative actions using the imperfective (was verb + ing) tense were less likely to be re-elected than if their actions were described using the perfective (verb + ed) tense. This is related to the fact that people may think that negative actions that are described using the imperfective tense are ongoing, whereas the perfective tense indicates clearly that the negative action is in the past. Linguistic variation has also been explored in more detail for texts in news reports (Henley et al., 2002) and in scientific reports written by students (Keys, 1999). These studies examined word specificity and their associated effects on the reader's perception and, for the scientific reports, the relationship between students' writing specificity and their understanding of scientific writing. Both studies found that linguistic variation in statements can affect the way a reader perceives the importance of a given situation, although this has yet to be explored in terms of texts on the conservation of species at risk. Considering that recovery strategy reports are a foundation for recovery for species at risk, they should be written to maximize the measurability, clarity and word specificity of statements.

To determine whether semantic uncertainty is a problem (and if so, to what extent) in the writing found in recovery strategies, we examined the 'recovery goal' and 'recovery objective' sections of all available provincial and federal recovery strategies in Canada. Canada is a relevant case study for such an investigation because its endangered species legislation is more recent than that in other large countries (e.g. the USA). We tested whether variations in word specificity, as a proxy for semantic uncertainty, of recovery statements were related to (1) the degree of endangerment, (2) the jurisdiction, or (3) the taxonomic grouping of species at risk. Our goal here is to evaluate whether the above factors influence word specificity of recovery strategies in Canada and to offer suggestions to improve the quality and effectiveness of those targets in terms of species' recovery.

\section{Methods}

We obtained the final versions of 213 federal and provincial (Ontario, Québec, Alberta, and British Columbia) recovery strategies for species at risk. Although Alberta and British Columbia do not have a provincial law to protect species at risk, they develop their own recovery strategies through species at risk programmes (Alberta Environment and Sustainable Resource Development, 2010; Ministry of Environment, 2011). In Alberta these documents are published and distributed by the Alberta Conservation Association and Alberta Sustainable Resource Development (Alberta Environment and Sustainable Resource Development, 2010; Alberta Conservation Association, 2012), whereas British Columbia develops its recovery strategies under the Canada-British Columbia Agreement on Species at Risk (Ministry of Environment, 2011).

Given that provinces have their own species at risk legislation and programmes, independent of the federal government, provincial recovery strategies were obtained separately from the online Species at Risk Act registry. Within each species recovery strategy we recorded the recovery goal and recovery objective statements, along with the specific listing status for the species. These statements identify what needs to be done to stop or reverse the decline of a species. More specifically, recovery goals state what it is hoped will be achieved, whereas recovery objectives describe the main actions and strategies needed to reach the recovery goals (Species at Risk Registry, 2010). The recovery goal and recovery objective statements for Québec were translated into English by a bilingual individual. We then analysed the statements for their overall specificity, using text analysis (Textalyser, 2004) that quantified both lexical density and readability values. In addition we examined each statement for the presence or absence of numerical population targets that would allow recovery success to be measured.

\section{Recovery strategies}

We analysed recovery strategies for four categories of increasing risk status: special concern, threatened, endangered and extirpated. Although recovery strategies are not generally written for species of special concern, they are a group for which management/recovery plans are written in some jurisdictions (e.g. Ontario) and species in this category (e.g. the monarch butterfly Danaus plexippus) are often numerous enough to offer rich population data that should allow for meaningful management targets. We assessed differences in recovery strategies based 
on taxonomic grouping, which we divided into those for vertebrates and those for invertebrates/plants. Finally, we recorded presence or absence of numerical indicators within each recovery goal and objective statement.

\section{Word specificity and explicit quantification}

Readability quantifies the elements that could affect the ease of comprehension of a given text. A text is readable to the extent that it provides the information that readers need, located where they can quickly find it, and in a form that they can easily use (Harrison \& Bakker, 1998). We used the Gunning Fog Index (GFI) to measure readability, ranking the degree of difficulty $1-20$, with 20 being the most difficult to understand (Textalyser, 2004). The index estimates the years of formal education needed to understand a text on first reading and is calculated based on counting sentences and non-complex and complex words based on syllable count. Texts intended for a broad audience generally need a GFI $<12$ (Harrison \& Bakker, 1998).

Lexical density is another way to quantify the degree of difficulty involved in understanding text. Texts are easier to read if they are written in a more oral style. Spoken discourse contains a lower ratio of lexical items (related to content) to grammatical items (related to sentence structure) than does written discourse. The more lexical terms a given sentence contains, the more lexically complex it becomes (Harrison \& Bakker, 1998; Keys, 1999). Lexical density is measured by the number of lexical terms per sentence and is expressed as a percentage (Textalyser, 2004).

Besides grammatical construction it is also important to examine whether the recovery goal statements and objective statements include any numerical terms (e.g. size of target population). Goals involving numerical components increase the specificity of recovery statements (Findlay et al., 2009; Mooers et al., 2007). Each recovery goal and objective statement was allocated a value of 1 or o based on whether a numerical indicator for population size targets was present (1) or absent (o).

\section{Statistical analyses}

We used a quasi-Poisson regression for overdispersed count data, using $R$ ( $\mathrm{R}$ Development Core Team, 2010), which is robust to assumptions of normality and homogeneity of variances. For all analyses we set $\alpha=0.05$. We used lexical density and readability as separate response variables and modelled each of them with the predictive variables of jurisdiction, taxonomic grouping and degree of endangerment of species at risk. We did not include any interaction terms in the models. We also used quasi-Poisson regression with the same predictive variables to model the responses

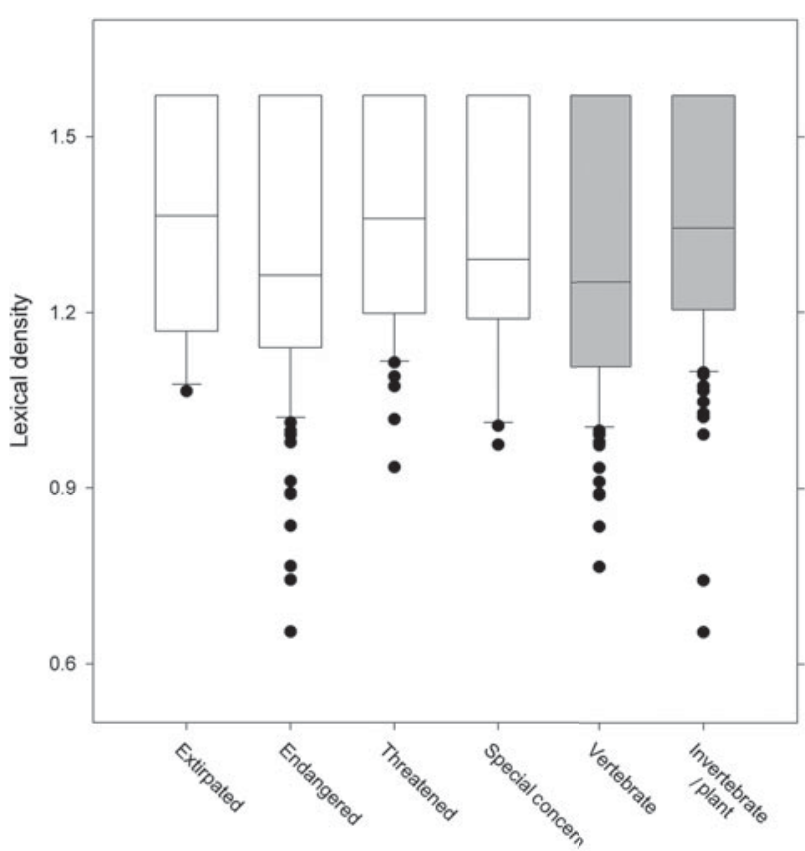

FIG. 1 Box plots (showing all outliers and median values) of the lexical density within recovery goal statements for each level of risk (white boxes) and whether the species was a vertebrate or invertebrate/plant (grey boxes).

of specificity of recovery goal and recovery objective statements separately. We then built quasi-Poisson models for both recovery goal and objective statements, and we used jurisdiction and level of endangerment as separate dependent variables in response to the predictive variable of presence or absence of population size targets.

\section{Results}

\section{Recovery goals}

The lexical density of recovery goals differed significantly between degree of endangerment $\left(t_{204}=2.18, \mathrm{P}<0.05\right.$; Fig. 1) and taxonomic groupings $\left(t_{208}=3.05, \mathrm{P}<0.01\right.$; Fig. 1). Recovery goals for invertebrates/plants and species in the threatened category have the highest lexical densities, which implies that the target statements for these are often long, complex and of low specificity. Lexical density of recovery goals did not differ between jurisdictions $(\mathrm{P}>0.1)$ although readability $\operatorname{did}\left(t_{209}=2.23, \mathrm{P}<0.05\right.$; Fig. 2). Readability of recovery goals also differed significantly between degrees of endangerment $\left(t_{208}=2.91, \mathrm{P}<0.01\right.$; Fig. 2). Recovery goals of recovery strategies in Ontario that pertain to threatened species had the highest readability values and thus were most difficult to understand. There were no differences in readability of recovery goals between taxonomic groupings $(\mathrm{P}>0.1)$. Differences in specificity for recovery goal statements were not related to whether discrete quantitative goals were provided. 


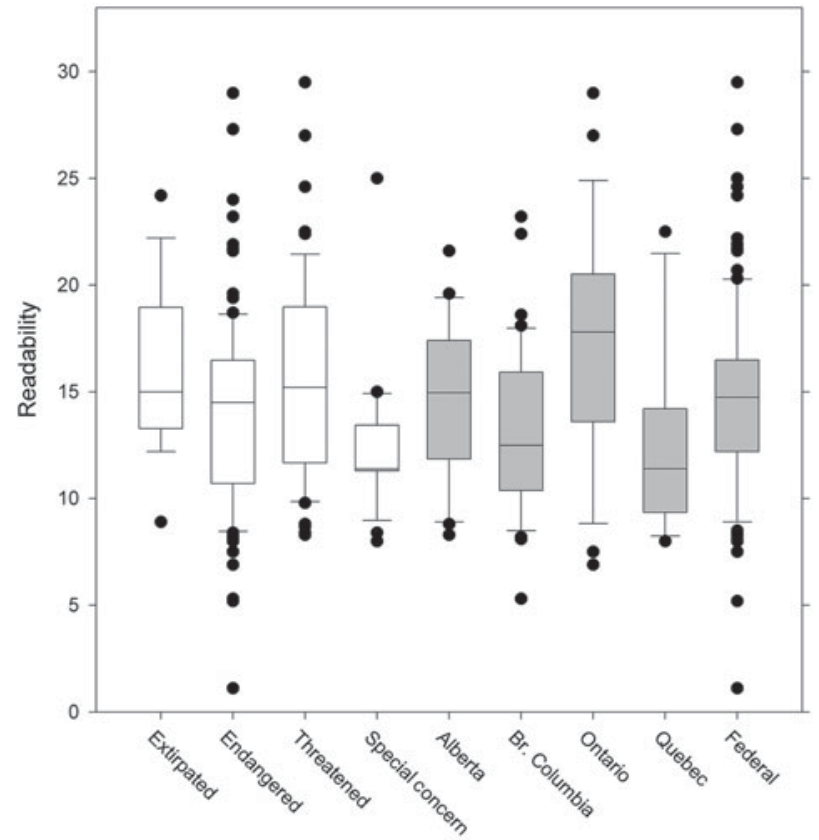

FIG. 2 Box plots (showing all outliers and median values) of the readability score for recovery goal statements for each level of risk (white boxes) and jurisdiction (grey boxes).

\section{Recovery objectives}

The specificity of recovery objectives differed only in terms of taxonomic grouping $\left(t_{166}=3.18, \mathrm{P}<0.01\right.$; Fig. 3$)$. Recovery objectives for invertebrate/plant species have higher lexical densities than those for vertebrate species, indicating that their statements were complex and low in specificity. Lexical densities of recovery objectives were not significantly different between jurisdictions or endangerment status $(P>0.1)$. There were no differences in specificity of recovery objectives in terms of presence/ absence of numerical indicators. Readability of recovery objectives did not differ in terms of jurisdiction, status of endangerment or taxonomic grouping $(\mathrm{P}<0.1)$.

Recovery objective statements were significantly more specific than recovery goal statements in terms of lexical density $\left(t_{376}=8.12, \mathrm{P}<0.001\right)$. There was no significant difference in readability when comparing recovery goals and objectives $(\mathrm{P}>0.1)$. Presence/absence of numerical indicators did not explain differences observed when used as a predictor variable for differences in specificity between jurisdiction and level of endangerment of recovery goal $(\mathrm{P}>0.1)$ and recovery objective $(\mathrm{P}>0.1)$ statements.

\section{Discussion}

Systematic patterns of semantic uncertainty exist in recovery strategy target statements for species at risk in Canada. Target statements for species in the threatened category were more lexically dense and difficult to read than

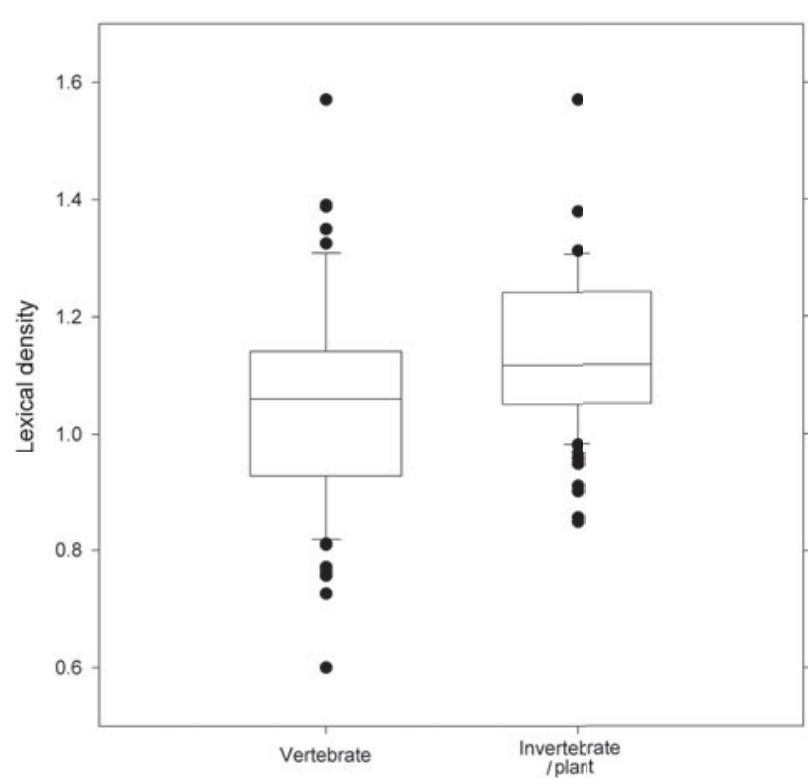

FIG. 3 Box-plots (showing all outliers and median values) of the lexical density within recovery objective statements for vertebrate species and for invertebrate/plant species.

those for species in the endangered or special concern categories. Recovery strategies for species in the threatened category may be subject to greater semantic uncertainty because these species are generally less abundant than special concern species (given that status assessments are based on rates of decline, number of populations, and decreased population indices; COSEWIC, 2011) but because they are typically more abundant and/or widespread than species in the endangered category they may have more imprecise population estimates (Cyr et al., 1992; Royle, 2004).

Lexical density, a measure of statement complexity, was lower for text on vertebrates (e.g. more charismatic species; Lundquist et al., 2002) than for text on plants and invertebrates. Vertebrates are assigned clearer recovery targets because there may be more empirical data available to guide population recovery and perhaps more resources available to study them (which may be representative of the greater value that society places on them). As a result of the focus on charismatic vertebrates, more data are accumulated for those species, resulting in better constructed recovery plans and their associated recovery goals and objectives (Lundquist et al., 2002). However, it is important to note that in some cases, such as annual plants and some cryptic or fluctuating species, a goal that specifies an amount of habitat could be an effective proxy to quantitative population targets. Other reasons for taxonomic bias in recovery statement word specificity may include policy considerations and conflicts of interest (Mooers et al., 2007).

There was little difference in the specificity of recovery goals and objectives among jurisdictions. The only exception to this is that recovery goals in strategies from Ontario 
tended to have high GFI values (making them more difficult to understand), which may correspond to a higher number of recovery strategies for plants and invertebrate species because of the disappearing Carolinian forest. With the removal of outliers with the highest GFI values (Fig. 2), which were both texts relating to plant species of the Carolinian forest, there were no significant differences in semantic uncertainty between jurisdictions $\left(t_{207}=1.79\right.$, $\mathrm{P}>0.05)$. It is reassuring that the jurisdictions we assessed were producing recovery targets with generally comparable specificity, especially as it is likely that many strategies for a given taxonomic group in a jurisdiction were written by the same person. In short, the semantic uncertainty that is more prevalent in recovery statements for threatened and invertebrate/plant species is not further complicated by regional differences or problems with non-independence associated with multiple strategies from a single author.

Recovery strategies in Canada would be more effective if the semantic uncertainty of recovery target statements for threatened and invertebrate/plant species were reduced. An example of how recovery strategy clarity is linked to population management is provided by Boersma et al. (2001), who quantified recovery plan effectiveness for species listed in the USA according to their population trends: improving, stable, declining, extinct or unknown. Species with revised recovery plans were associated with an improvement in status in the USA compared with species that had unrevised plans. We speculate that the improvement of status for these species may be because of the addition of new information that improves the effectiveness of the recovery plans. Such new information should translate into new recovery goals and criteria that have less semantic uncertainty because they are more clearly linked to the species' biology by incorporating recovery metrics such as population size, range size and habitat quality. These connections between recovery goals and a species' ecological data significantly improve recovery plan effectiveness, and thus ultimately species status. Perhaps because success cannot be defined when goals simply invoke general and vague statements of ecology or conservation biology, such recovery strategies are less likely to succeed.

The USA-based results of the study by Boersma et al. (2001) are encouraging for Canada and the construction/ revision of recovery strategies; however, whether a similar relationship between recovery strategy specificity and status improvement exists in Canada or its provinces is unknown. The US Endangered Species Act has been in place since 1973, much longer than the Canadian Species at Risk Act, implemented in 2002, and few species covered by the latter have had their status changed. The Species at Risk Act is still developing, however, so we can consider the criticism that the ESA has received over time and resolve the semantic biases detected in this study, thereby improving recovery strategies to benefit the recovery of listed species in Canada.
To improve semantic uncertainty through better use of science and information we make the following recommendations for the improvement and development of future recovery strategies: (1) maintain awareness that species rarity seems to be associated with recovery plan specificity, so that extra care needs to be taken in ensuring recovery strategies are specific for species in the special concern and threatened categories, (2) increase the amount of data collection and funding for less charismatic species, to rectify the differential effort currently being applied to vertebrates vs invertebrates and plants, and (3) increase the number of plan revisions, to incorporate the most up-todate scientific information and thus keep the plans relevant.

\section{Acknowledgements}

We thank Philina English, Barbara Frei, Chris Kyle, Val von Zuben and Hazel Wheeler for discussion and review that improved this article, and Marco Festa-Bianchet and an anonymous reviewer for constructive comments. The work presented here was made possible by a collaborative internship programme between Trent University and the Ontario Ministry of Natural Resources. We are grateful for the public access to recovery strategies and associated documents at both the federal (Canada) and provincial (Ontario, Québec, Alberta, and British Columbia) levels.

\section{References}

Akçakaya, H.R., Ferson, S., Burgman, M.A., Keith, D.A., MACE, G.M. \& Todd, C.R. (2000) Making consistent IUCN classification under uncertainty. Conservation Biology, 14, 1001-1013.

Alberta Conservation Association (2012) Wildlife Program. Http://www.ab-conservation.com/go/default/index.cfm/programs/ wildlife/ [accessed 25 July 2012].

Alberta Environment and Sustainable Resource Development (2010) Species at Risk. Http://srd.alberta.ca/ FishWildlife/SpeciesAtRisk/Default.aspx [accessed 30 September 2010].

Boersma, P.D., Kareiva, P., Fagan, W.F., Clark, J.A. \& Hoekstra, J.M. (2001) How good are endangered species recovery plans? BioScience, 51, 643-649.

COSEWIC (Committee on the Status of Endangered Wildlife in Canada) (2011) COSEWIC's Assessment Process and Criteria. Http://www.cosewic.gc.ca/eng/scto/ assessment_process_e.cfm [accessed 21 June 2012].

Cyr, H., Downing, J.A., Lalonde, S., Baines, S.B. \& Pace, M.L. (1992) Sampling larval fish populations-choice of sample number and size. Transactions of the American Fisheries Society, 121, 356-368.

Elphick, C.S., Reed, J.M. \& and Bonta, J.M. (2001) Correlates of population recovery goals in endangered birds. Conservation Biology, 15, 1285-1291.

Fausey, C.M. \& Matlock, T. (2011) Can grammar win elections? Political Psychology, 32, 563-574.

Findlay, C.S., Elgie, S., Giles, B. \& Burr, L. (2009) Species listing under Canada's Species at Risk Act. Conservation Biology, $23,1609-1617$. 
Gerber, L.R. \& Hatch, L.T. (2002) Are we recovering? An evaluation of recovery criteria under the U.S. Endangered Species Act. Ecological Applications, 12, 668-673.

Harrison, S. \& BaKker, P. (1998) Two new readability predictors for the professional writer: pilot trials. Journal of Reading Research, 21, 121-138.

Henley, N.M., Miller, M.D., Beazley, J.A., Nguyen, D.N., Kaminsky, D. \& Sanders, R. (2002) Frequency and specificity of referents to violence in news reports of anti-gay attacks. Discourse and Society, 3, 75-104.

Keys, C.W. (1999) Language as an indicator of meaning generation: an analysis of middle school students' writing discourse about scientific investigations. Journal of Research in Science Teaching, 36, 1044-1061.

Lundquist, C.J., Diehl, J.M., Harvey, E. \& Botsford, L.W. (2002) Factors affecting implementation of recovery plans. Ecological Applications, 12, 713-718.

Ministry of Environment (2011) Recovery Planning in British Columbia. Http://www.env.gov.bc.ca/wld/recoveryplans/rcvryı.htm [accessed 25 July 2012].

Mooers, A.O., Doak, D.F., Findlay, C.S., Green, D.M., Grouios, C., Manne, L.L. et al. (2010) Science, policy, and species at risk in Canada. Bioscience, 60, 843-849.

Mooers, A.O., Prugh, L.R., Festa-Bianchet, M. \& Hutchings, J. A. (2007) Biases in legal listing under Canadian endangered species legislation. Conservation Biology, 21, 572-575.

R Development Core Team (2010) R: A Language and Environment for Statistical Computing. R Foundation for Statistical Computing, Vienna, Austria.
Royle, J.A. (2004) N-mixture models for estimating population size from spatially replicated counts. Biometrics, 60, 108-115.

Schultz, C.B. \& Gerber, L.R. (2002) Are recovery plans improving with practise? Ecological Applications, 12, 641-647.

Schwartz, M.W. (2008) The performance of the Endangered Species Act. Annual Review of Ecology, Evolution, and Systematics, $39,279-299$.

Semlitsch, R.D. (2001) Critical elements for biologically based recovery plans of aquatic breeding amphibians. Conservation Biology, 16, 619-629.

Species at Risk Registry (2010) Recovery Strategies. Http://www. sararegistry.gc.ca/sar/recovery/recovery_e.cfm [accessed 30 September 2010].

Textalyser (2004) Textalyser. Http://textalyser.net/index.php? lang=en\#analysis [accessed 21 November 2010].

Wilcove, D.S., McMillan, M. \& Winston, K.C. (1993) What exactly is an endangered species? An analysis of the Endangered Species List, 1985-1991. Conservation Biology, 7, 87-93.

\section{Biographical sketches}

Elysabeth Théberge's interests and work are focused on conservation biology, specifically the conservation of aquatic ecosystems. Her most recent work is on benthic and running-water ecology. Joseph J. Nocera focuses on research into species-at-risk and their management, with a particular interest in how animal behaviour can be used and studied to further conservation efforts. $\mathrm{He}$ is investigating the causes and consequences of population declines in aerial insectivores and grassland birds. 